\title{
Estructura y dinámica de las interrelaciones en el espacio geográfico
}

\author{
Reseña de: Santos, Milton (2000), La naturaleza del espacio, \\ ARIEL, España.
}

Una de las categorías medulares dentro de la geografía es el espacio, cuyas observaciones y resultados parten de un sistema descriptivo y cualitativo de las relaciones sociales y su entorno. Aunque la relevancia de estudiar al espacio no constituye un motivo reciente, resulta importante analizar qué otros elementos, categorías o factores se ofrecen en cada nuevo intento exploratorio, pues ello implica repensar las interrelaciones y las prácticas territoriales propias de la dinámica social. Evidentemente, esto significa una relación recíproca entre los procesos de transformación de las estructuras sociales y el individuo con relación al espacio.

En el ejercicio de analizar el corpus de la geografía, van apareciendo diferentes discusiones sobre el objeto, método, naturaleza, categorías de estudio y definiciones que le conciernen. Los esfuerzos interpretativos van desde la búsqueda de conceptos, asociaciones y aplicaciones sobre la dinámica social, hasta interrogarse sobre el papel del espacio-tiempo y el lugar, en el proceso de construcción de una base para la geografía. Obviamente, uno de los desafíos es poder analizarla de manera autónoma, pero no independiente de los elementos de consistencia, adecuación y pertinencia de su contexto.

Bajo las consideraciones anteriores, el libro La naturaleza del espacio de Milton Santos, recientemente publicado, enfrenta a los interesados en el análisis geográfico a una serie de contradicciones conceptuales inherentes a la disciplina, y que dan pauta para reconsiderar viejos debates sobre la configuración social del espacio, su funcionamiento y estructura, haciendo la debida aclaración de que la definición o pertinencia de cada concepto variará de acuerdo con el momento histórico que le acompañe. Esto también implica poner en claro que en el espacio geográfico se fusionan materialidades y acciones, así como condiciones locales y globales, soportadas por las redes que integran y disuelven las acciones de la sociedad. 
De este modo, Milton Santos aborda uno de los problemas principales de la geografía: identificar la naturaleza del espacio y encontrar las categorías de análisis que permitan estudiarlo. Este proyecto supone encontrar aquellos conceptos, asociaciones y aplicaciones que puedan operar sobre la dinámica social; lo que constituye un ejercicio de análisis complejo, cuya intención intenta revelar aquellas categorías que, en muchos de los casos, aparecen separadas del corpus de la geografía, tales como la conocida unión entre espacio-tiempo, la importancia del lugar y el espacio en el proceso social y, finalmente, la propia delimitación y pertinencia de la geografía en el momento actual.

Santos parte de la noción de espacio como aquel conjunto indisoluble de sistemas de objetos y sistemas de acciones, en el que confluyen categorías analíticas como: el paisaje, la configuración territorial, la división territorial del trabajo, el espacio producido o productivo, las rugosidades y las formas contenidas. De esta manera, el autor nos incita a reconsiderar el debate sobre problemas como la región, el lugar, las redes, las escalas, el orden local y global. Asimismo, hace patente el reconocimiento de procesos básicos externos al espacio como: la técnica, la acción, los objetos, la norma y los acontecimientos, la universalidad y la temporalidad, la idealización y la objetivación, los símbolos y la ideología.

Cabe señalar que para Santos, el espacio adquiere contenido a partir del reconocimiento de los vínculos entre el individuo y la sociedad; parte de la idea de que es en el espacio donde confluyen relaciones de carácter funcional, de interdependencia, de selección, de reproducción, de sustitución o de cambio, cuya actuación se refleja en diferentes escalas, niveles y tiempos. En este sentido, la dinámica socioterritorial está funcionalmente ligada a los cambios propios del espacio, es decir, a las manifestaciones, procesos y articulaciones, de los sistemas sociales. El espacio se recrea dinámica y permanentemente en convivencias trascendentes y efímeras, cuyas formas, contenidos, reglas, funcionamientos, dirección y capacidad se sostienen bajo procesos socio-espaciales en movimiento donde las posibilidades de permanencia dependen de las potencialidades y capacidad para sostener procesos locales y globales, según su propia funcionalidad y dialéctica.

La intención explícita de Milton Santos es que su libro pueda ser una contribución geográfica a la producción de una teoría social crítica, al mismo tiempo que pueda ser un punto de partida 
para la producción de ideas y nuevas interpretaciones sobre el espacio geográfico (Santos, 2000: 20). Para ello identifica cuatro momentos. En el primero, Una ontología del espacio: nociones originarias, donde trabaja con las ideas ontológicas del espacio: la técnica, el tiempo y la intencionalidad, materializados en los objetos y acciones. Asimismo, da pauta para analizar la técnica como una totalidad, lo que obliga a analizarla en el contexto histórico del territorio y en las relaciones desiguales de convivencia.

En un primer esbozo, aborda la importancia de las técnicas y su desempeño en las relaciones con los hechos sociales. La participación de los sistemas técnicos en la dinámica social ha sido determinante, pues tanto la infraestructura como las transformaciones de uso de las necesidades prácticas han estado supeditadas a los procesos productivos, culturales y territoriales de una red de técnicas. Su análisis no debe limitarse a sus aplicaciones netamente funcionales, más bien debe situarse en la comprensión de las múltiples relaciones geográficas que genera y en el "estudio desde dentro del espacio, y no sólo externo a esa realidad social, que permitiera una interpretación de la forma en que el territorio ha sido modificado y transformado con las nuevas presencias técnicas". (p. 33).

Más adelante, Santos reconoce que el espacio está formado por un conjunto indisoluble, solidario y también contradictorio, de sistemas de objetos y sistemas de acciones no considerados aisladamente, sino como el contexto único en el que se realiza la historia. Por un lado, los sistemas de objetos condicionan la forma en que se dan las acciones y, por otro, el sistema de acciones lleva a la creación de objetos nuevos o se realiza sobre objetos preexistentes. Así, el espacio se encuentra en una dinámica de transformación constante. (pp. 54-55).

El autor aclara que el tema central de la geografía no son los objetos ni las acciones por separado, sino objetos y acciones tomados en conjunto. Donde se recuerda que los resultados de la acción humana no dependen únicamente de la racionalidad de la decisión y de la ejecución, sino también, de la naturaleza humana y del carácter humano del medio. Donde un nuevo sistema de objetos responde al surgimiento de cada nuevo sistema de técnicas, donde existe también un nuevo ordenamiento de objetos ( $\mathrm{p}$. 80). Cuando la sociedad actúa sobre el espacio, no lo hace sobre los objetos como realidad física, sino como realidad social, for- 
mas-contenido, es decir, objetos sociales ya valorizados a los cuales la sociedad busca ofrecer o imponer un nuevo valor (p. 91).

En el segundo momento, titulado La producción de las formas-contenido se retoma la cuestión ontológica, considerando el espacio como forma-contenido. En esta parte del libro se intenta reconocer cómo el proceso de transformación de una totalidad va sufriendo modificaciones en su estructura a partir de la existencia dinámica de la sociedad, de sus acciones, de las propias configuraciones territoriales y materiales del espacio, y finalmente, de la propia división del trabajo. Así también, se reconoce la importancia del tiempo como importante motor en la cristalización de los procesos de globalización.

En este mismo apartado se reconoce que es necesario retomar el concepto de totalidad, revisar sus formas de apariencia, reconocer su metamorfosis y su proceso, y analizar sus implicaciones con la propia existencia del espacio (pp. 96-97). El movimiento de la totalidad para existir objetivamente es un movimiento dirigido a su especialización, que es también particularización (p. 105). Por ello, en cada nueva división del trabajo, en cada nueva transformación social hay, paralelamente para los fabricantes de significados, una exigencia de renovación de las ideologías y de los universos simbólicos, al mismo tiempo que, para los demás, se hace posible el entendimiento del proceso y la búsqueda de un sentido.

El autor señala también que en cada uno de sus momentos, el proceso social involucra una redistribución de sus factores. Y esa redistribución no es diferente a las condiciones preexistentes, es decir, a las formas heredadas, provenientes de momentos anteriores. Las formas naturales y el medio ambiente construido se incluyen entre esas formas heredadas. Se señala adicionalmente que, en cada lugar el tiempo actual se enfrenta con el tiempo pasado, cristalizado en formas, donde el trabajo ya hecho se impone sobre el trabajo por hacer, es decir, que la actual distribución territorial del trabajo descansa sobre las anteriores divisiones territoriales del mismo (pp. 118-119).

La comprensión de los lugares en su situación actual y en su evolución depende de la consideración del eje de las sucesiones y del eje de las coexistencias, donde se reconoce que el espacio es el que reúne a todos, con sus múltiples posibilidades, que son posibilidades diferentes de uso del espacio (del territorio) relacionadas con posibilidades diferentes de uso del tiempo (pp. 134-135). En cada momento, hay siempre un mosaico de subes- 
pacios, cubriendo enteramente la superficie de la Tierra y cuyo diseño es proporcionado por el curso de la historia: la escala deja de ser una noción geométrica para ser condicionada por el tiempo (p. 141).

En el tercer momento, que abarca los capítulos 7 al 13, y se titula Por una geografía del presente, las nociones anteriormente establecidas son revisadas a la luz del presente histórico, para aprehender la constitución actual del espacio y las condiciones del mundo. Aparecen elementos como sistemas técnicos, unicidades, fijos y flujos, horizontalidades y verticalidades, espacios de racionalidad, entre otros. De forma inicial, en los capítulos séptimo y octavo destaca que las características de la sociedad y del espacio geográfico, en un momento de su evolución, están en relación con un determinado estado de las técnicas.

Por lo tanto, el conocimiento de los sistemas técnicos sucesivos es esencial para la comprensión de las diversas formas históricas de estructuración, funcionamiento y articulación de los territorios, desde los albores de la historia hasta la época actual. Cada periodo es portador de un sentido, compartido por el espacio y por la sociedad, representativo de la manera en que la historia realiza las promesas de la técnica (p. 145). El autor reconoce que las manifestaciones actuales de unicidad técnica, de tiempo (a su vez como motor de la vida económica) son la base del fenómeno de globalización y de las transformaciones contemporáneas del espacio geográfico (p. 159).

En los capítulos noveno y décimo se menciona que las características del sistema técnico actual son: la universalidad y autoexpansión, la vida sistémica, la concreción, el contenido de información y la intencionalidad (p. 180). Donde el territorio como un todo se convierte en un dato de esa armonía forzada entre lugares y agentes allí instalados, en función de una inteligencia mayor situada en los centros motores de la información. Esto provoca que las relaciones entre sociedad y naturaleza se vayan sustituyendo de un medio natural por un medio cada vez más artificial, es decir, sucesivamente instrumentalizado por esa misma sociedad (p. 199). Lo anterior responde a la sombra de la presente democracia de mercado que vivimos, en la que el territorio es soporte de redes que transportan las verticalidades, es decir, reglas y normas egoístas y utilitarias, mientras que las horizontalidades tienen en cuenta la totalidad de los actores y de las acciones (p. 219). 
En los capítulos 11, 12 y 13 se incorporan diversos elementos. En principio, se dice que las redes serían incomprensibles si sólo las vislumbrásemos a partir de sus manifestaciones locales o regionales. Para ello, parte de reconocer que las redes son un vehículo de un movimiento dialéctico que, por un lado, opone el territorio y el lugar al mundo y, por otro, enfrenta el lugar al territorio tomado como un todo (pp. 228-229). Hoy, las segmentaciones y particiones en el espacio sugieren que se admitan, al menos, dos dimensiones. Por un lado, hay extensiones formadas de puntos que se agregan sin discontinuidad, como en la definición tradicional de la región (horizontalidades). Por otro lado, existen puntos en el espacio que, separados unos de otros, aseguran el funcionamiento global de la sociedad y de la economía (verticalidades) (p. 240).

Otro punto destacable es saber si es lícito hablar de una racionalidad del espacio geográfico, del mismo modo que nos referimos a la racionalidad o a la racionalización de otras facetas de la realidad social (p. 245). El hecho de que la producción limitada de racionalidad esté asociada con una amplia producción de escasez conduce a los actores -que están fuera del círculo de la racionalidad hegemónica- al descubrimiento de su exclusión y a la búsqueda de formas alternativas de racionalidad, indispensables para su supervivencia. La racionalidad dominante y ciega acaba produciendo sus propios límites (p. 263).

En el cuarto momento, que comprende los capítulos 14 y 15 , el autor destaca que el reconocimiento de racionalidades convergentes, frente a la racionalidad dominante, refleja las nuevas perspectivas de método y de acción, autoriza cambios de perspectiva en cuanto a la evolución espacial y social, y aconseja cambios en la epistemología de la geografía y de las ciencias sociales como un todo. Asimismo subraya que el surgimiento de un medio técnico-científico-informacional ha provocado una nueva etapa en el proceso secular de la racionalización.

En los ambos capítulos se aprecia que el espacio se ofrece al conjunto de los hombres que en él actúan como un conjunto de potencialidades de valor desigual, cuyo uso tiene que ser disputado a cada instante, en función de la fuerza de cada uno (p. 270). Cuanto más inestable y sorpresivo es el espacio, tanto más sorprendido será el individuo y tanto más eficaz la operación de descubrimiento. La conciencia por el lugar se superpone a la conciencia en el lugar. La noción de espacio desconocido pierde la 
connotación negativa y gana un acento positivo, que proviene de su papel en la producción de la nueva historia (p. 281).

Al respceto, Milton Santos también menciona que las redes son mixtas, que incluyen materialidad y acción. En este contexto, la red técnica mundializada actual es instrumento de la producción, de la circulación y de la información mundializadas. En este sentido, las redes son globales y, de esa manera, transportan lo universal a lo local. Sin embargo, las redes también son locales y por esa naturaleza constituyen las condiciones técnicas del trabajo directo de la misma manera que las redes globales aseguran la división del trabajo y la cooperación, mediante las instancias no técnicas del trabajo: la circulación, la distribución y el consumo (p. 285). Señala también que cada lugar es, al mismo tiempo, objeto de una razón global y de una razón local que conviven dialécticamente (p. 290).

Podemos señalar, finalmente, que las mezclas, innovaciones e interrelaciones dadas en el espacio, son categorías materializadas en los estudios regionales, en las que conviven expresiones del pasado y propias del momento. Las tensiones entre la permanencia del pasado y el surgimiento de la velocidad en los cambios técnicos y conceptuales provocan que los procesos de envejecimiento se aceleren o dilaten de forma diferenciada en los territorios y, obviamente, en sus acciones.

Milton Santos nos invita a reflexionar sobre la estructura de las interrelaciones en el espacio geográfico, en el que se sostienen nuevas formas de conflictos, movimientos y decisiones, lo que da pauta a constituir una forma de defensa o resistencia, pero al mismo tiempo una forma de incorporación o no segregación. Así, el reconocimiento de categorías de análisis internas y externas son fundamentales para entender los actuales procesos geográficos.

Celia Hernández Diego Facultad de Planeación Urbana y Regional, Universidad Autónoma del Estado de México Correo-e: chd13@hotmail.com 